\title{
COMPACT RECONNAISSANCE IMAGING SPECTROMETER FOR MARS (CRISM)
}

\author{
Mr.Kaiem Frink, Dr. Linda Hayden, Dr. Malcom LeCompte \\ Center of Excellence in Remote Sensing Education and Research \\ Elizabeth City State University \\ Elizabeth City, North Carolina, USA
}

\begin{abstract}
The Compact Reconnaissance Imaging Spectrometer for Mars CRISM (CRISM) carried aboard the Mars Reconnaissance Orbiter (MRO), is the first visible-infrared spectrometer to fly on a NASA Mars mission. CRISM scientists are using the instrument to look for the residue of minerals that form in the presence of water: The 'fingerprints' left by evaporated hot springs, thermal vents, lakes or ponds. With unprecedented clarity, CRISM is mapping regions on the Martian surface at scales as small as 60 feet (about 18 meters) across, when the spacecraft is 186 miles (300 kilometers) above the planet. CRISM is reading 544 'colors' in reflected sunlight to detect certain minerals on the surface, including signature traces of past water. CRISM alone will generate more than 10 terabytes of data, enough to fill more than 15,000 compact discs. Given that quantity of data being returned by MROCRISM, this project partners with Johns Hopkins University (JHU) Applied Physics Laboratory (APL) scientists of the CRISM team to assist in the data analysis process. The CRISM operations team has prototyped and will provide the necessary software analysis tools. In addition, the CRISM operations team will provide reduced data volume representations of the data as PNG files, accessible via a web interface without recourse to specialized user tools. The web interface allows me to recommend repeating certain of the CRISM observations as survey results indicate, and to enter notes on the features present in the images.
\end{abstract}

Keywords-component; Digital Divide, Mathematics, Space Science,

Mars, Rust, Remote Sensing

\section{INTRODUCTION}

The Center of Excellence in Remote Sensing Education and Research (CERSER) Compact Reconnaissance Imaging Spectrometer For Mars (CRISM) website was managed by NASA, APL, JHU, JPL. The objective of this research project was to identify the chemical properties that are present on Mars. The website allowed for a web interface where data set could be viewed via internet connection. The goal of this project was to develop a new scripting file utilizing current file type, and the My Structure Query
Language (MySQL) to process these images. The data set had several steps that require detail instruction toward implementation.

During the testing and prototype of this research collaborators denoted that a Mathematical algorithm would be most important and useful in analyzing several 1,000 files of data. Where each file had five files that were associated with just one Master file. The algorithm that was developed and tested with Mathematica and Mathlab was known as Deese Algorithm. This simple however effective algorithm would analyze a given data set. The image would be grouped into small quadrants sets where mathematical mapping could be implemented and used to identify noise in several data sets.

The public outreach aspect of the paper allowed for Elizabeth City State University to assist in closing the Digital Divide in a rural community. As a result of this event ECSU participate in the National GIS DAY at Elizabeth City Middle School where we conducted Geographical Information Systems workshops and GPS training for the students to expose local area student to the higher order thinking and many unknown science fields in preparations of building the next generation.

The CRISM project has contributing to meaningful collaborations at Minority Serving Institution in the demographics of Historical Black College or University. Where there are many of historical events taking place while contributing the overall good of the human species as a co inhibitor of planet earth.

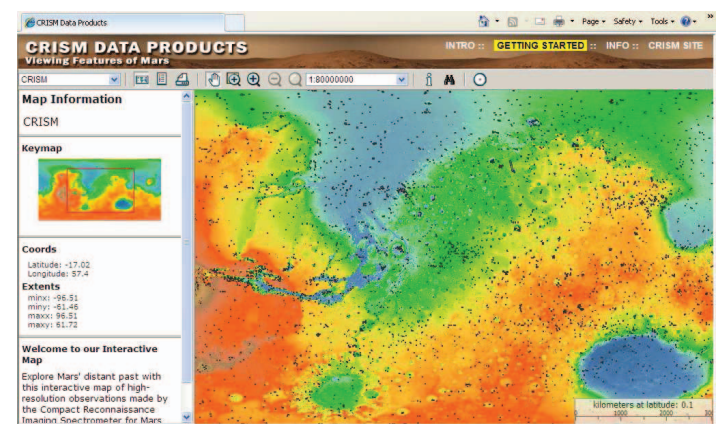

Figure: 1

CRISM Data Set with Latitude and Longitude coordinates 


\section{References:}

[1] On Minorities in Science: Examining the Role of Mentorship Programs in Earth Sciences

[2] Branch, Benjamin, Brittany Maybin and Kaiem Frink, An Informatics Pipeline, Earth and Space Science Informatics Workshop, Developing the Next Generation of Earth and Space Science Informatics: Technologies and the People That Will Implement Them, University of Maryland, Baltimore County, August 3, 4, and 5, 2009

[3] K.Frink, L. Hayden, and M. LeCompte, "CRISM", AGU Toronto Space Informatics, May 2009. 